\title{
Correspondence
}

\section{CREAM: a database for chemotherapy resistance-associated miRSNP}

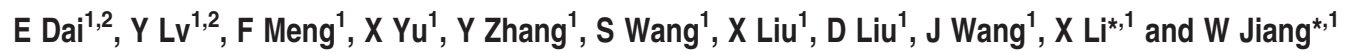 \\ Cell Death and Disease (2014) 5, e1272; doi:10.1038/cddis.2014.236; published online 29 May 2014 \\ Subject Category: Cancer
}

\section{Dear Editor,}

In current cancer treatment, fighting against cancer with cytotoxic drug is often challenged by the emergence of chemotherapy resistance, because the patients are usually treated by standard protocols without considering individual response to chemotherapy. ${ }^{1}$ As a promising field of personalized therapy of cancer, microRNA pharmacogenomics promotes to understand different individual responses to certain drugs and provides reasonable reference for clinical treatment. ${ }^{2,3}$ However, it is still lacking comprehensive resource to discover and deposit such chemotherapy resistance-associated miRSNPs (CRmiRSNPs). Therefore, we constructed an omnibus repository named CREAM (Chemotherapy ResistancEAssociated MiRSNP), which furnishes a user-friendly interface for a convenient retrieval of CRmiRSNP. The CREAM is freely available at http://bioinfo.hrbmu.edu. cn/CREAM/.

In CREAM, two types of CRmiRSNP (SNP that locates in the precursor of microRNA or $3^{\prime}$ UTR of microRNA target gene transcript, in which all these SNP, microRNA or gene are associated with chemotherapy response for the same compound) were mainly focused on, because both of these two CRmiRSNPs have been proven to influence the function of microRNA and may convert a sensible tumor cell into a resistant one by changing the expression of microRNA or their targets. ${ }^{2}$ Through utilizing genomic location and microRNA regulatory information, we have identified 150 CRmiRSNPs (Supplementary Figure 1a) across 1164 compounds from the chemotherapy response data as well as SNP genotyping, gene expression and microRNA expression profiles (more details in the 'Technical Implementation' section of Supplementary Information).

As an efficient search platform, CREAM supports four types of query (including compound, gene, microRNA and
SNP) for a convenient retrieval of each record, which not only offers the complete details of CRmiRSNP we have discovered, but also extends to display each compound with the elaborative descriptions and genomic distribution maps of chemotherapy resistance-associated molecules (including SNP, gene and microRNA). For instance, an interested compound can be queried to access which miRSNPs, genes, microRNAs and SNPs are associated with the chemotherapy response of this compound. Additionally, users can further visualize the chromosomal location and statistical significance of chemotherapy resistance-associated molecules for the queried compound (Supplementary Figure 1b). Finally, full entries in CREAM can be easily downloaded for systematic analysis (more details in the 'Practical Example' section of Supplementary Information).

Several miRSNP-related databases, centered on miRSNP's location and function, have been built recently. ${ }^{4,5}$ However, the resource that discovers the link between miRSNP and chemotherapy resistance has not been reported. The database of CREAM may contribute to systematic analysis of abundant chemicals (in this version it includes 1164 chemicals) in basic drug discovery, and the proposed strategy of identifying CRmiRSNP is in favor of precise selection of proper treatment in clinical cancer therapy. With constant expansion and improvement, we hope this resource will become a bridge between the laboratory investigation and clinical practice in the field of chemotherapy resistance research and stimulate further interest on the role of interactions among multi-level molecules in the individual chemoresponse network.

\section{Conflict of Interest}

The authors declare no conflict of interest.

\footnotetext{
${ }^{1}$ College of Bioinformatics Science and Technology, Harbin Medical University, Harbin, China

*Corresponding author: W Jiang or X Li, College of Bioinformatics Science and Technology, Harbin Medical University, 194 Xuefu Road, Harbin 150081, China. E-mail: jiangwei@ hrbmu.edu.cn (WJ) or lixia@ hrbmu.edu.cn (XL)

${ }^{2}$ The authors wish it to be known that, in their opinion, the first two authors should be regarded as joint First Authors.
} 
Acknowledgements. This work was supported by the National Natural Science Foundation of China (30900837) and the Foundation for University Key Teacher of the Education Department of Heilongjiang Province (1252G037)

1. Shekhar MP. Curr Cancer Drug Targets 2011; 11: 613-623.

2. Mishra PJ, Banerjee D, Bertino JR. Cell Cycle 2008; 7: 853-858.

3. Bertino JR, Banerjee D, Mishra PJ. Pharmacogenomics 2007; 8: 1625-1627.

4. Liu C et al. BMC Genomics 2012; 13: 661

5. Bhattacharya A, Ziebarth JD, Cui Y. Nucleic Acids Res 2013; 42: D86-D91.
Cell Death and Disease is an open-access journal published by Nature Publishing Group. This work is licensed under a Creative Commons Attribution-NonCommercialShareAlike 3.0 Unported License. The images or other third party material in this article are included in the article's Creative Commons license, unless indicated otherwise in the credit line; if the material is not included under the Creative Commons license, users will need to obtain permission from the license holder to reproduce the material. To view a copy of this license, visit http://creativecommons.org/licenses/ by-nc-sa/3.0/

Supplementary Information accompanies this paper on Cell Death and Disease website (http://www.nature.com/cddis) 